Instituto Internacional de Investigación y Desarrollo Tecnológico Educativo INDTEC, C.A.

DOI: https://doi.org/10.29394/scientific.issn.2542-2987.2017.2.3.14.260-275

OAI-PMH: http://www.indteca.com/ojs/index.php/Revista Scientific/oai

\title{
Responsabilidad Social del Personal Directivo - Docente en el Fortalecimiento del Entorno Comunitario
}

\author{
Autora: Marilú del Valle Parra Rojas \\ Universidad Nacional Experimental Rafael María Baralt, UNERMB \\ mvlu27@gmail.com \\ Zulia, Venezuela
}

\section{Resumen}

La presente investigación tuvo como propósito comprometer socialmente al personal directivo- docente en el fortalecimiento del entorno comunitario de la Unidad Educativa Nacional Héroe Nígale del municipio Valmore Rodríguez del Estado Zulia. Para ello se realizó una revisión de los documentos relacionado con la tendencia teórica sobre la Responsabilidad y el Compromiso Social. Para el desarrollo de este estudio se llevó a cabo una investigación acción participativa, basándose en el modelo de Elliont (2007), la investigación se ejecutó con el personal directivo- docente, así como también con los padres y representantes. La información se obtuvo por medio de entrevistas, registro de campo, grupo focal. La acción transformadora se realizó con la ejecución de los planes de acción que permitieron lograr los resultados pertinentes para comprometer de manera responsable y socialmente a todos los actores del hecho educativo y lograr así la calidad educativa que exige el Ministerio del Poder Popular para la Educación. Por otra parte, se pudo concluir que tanto el director como el docente es aquel que está comprometido con la educación venezolana, y son quienes proyectan a la comunidad el desarrollo de una sociedad formando ciudadanos para un país, capaces de ser, de aprender, de vivir y convivir con otros.

Palabras clave: actores; educativo; responsables; compromiso; acción. 


\title{
Social Responsibility of Directive - Teaching Personnel in the Strengthening the Community Environment
}

\begin{abstract}
This research was aimed at socially committing the directive - teaching personnel in the strengthening of the community environment in "National Educational Unit Heroe Nigale" from Valmore Rodriguez municipality in Zulia state. To do this, a revision of the documents related to the theoretical trend on Responsibility and Social Commitment was made. For the development of this study, a participatory action-research was carried out, based on model Elliont (2007), the investigation was conducted with the directive-teaching personnel as well as parents and guardians. The information was obtained through interviews, field record, and focus group. The transformational action was made with the application of action plans that allowed to accomplish pertinent results to commit, socially and responsibly, all performers of the educational process and thus accomplish the quality of education required by the People's Ministry for Education. On the other hand, we can conclude that both, the director and the teacher, are the ones committed to the Venezuelan education, they're the ones who project to the community the development of a society, forming citizens for a country, capable of being, learning, living and coexisting with others.
\end{abstract}

Keywords: performers; educational; responsible; commitment; action.

Date Received: 09-08-2016

Date Acceptance: 18-09-2016 


\section{Introducción}

Las reformas educativas han traído consigo cambios de paradigmas a nivel social, las misma han significado una revolución en las prácticas de acción participativa. Estas proponen cambios radicales en función de una educación para asumir una actitud positiva hacia el compromiso social. Significa, exigencias en la transformación de los entornos educativos, la cual implica la promoción de la toma de conciencia que sea posible asimilar los nuevos cambios que acuña el hombre desde su cultura.

En este sentido, la responsabilidad social de los actores del proceso educativo están en que todos deben asumir el compromiso con las organizaciones educativas y la misma comienza cuando el director y los docentes cumplen con su rol para responder a los planes conferidos por políticas, planes, programas y proyectos referidos en el plan de la nación consagrados en la Ley Orgánica de Educación, que son ineludible a la formación del ser humano y emanados por el sistema educativo, es decir implica llevar la escuela hacia una cultura de participación, para transformar las realidades de las comunidades brindando la formación académica el apoyo y la orientación para el mejoramiento de problemas que confrontan durante la gestión escolar.

Dentro de esta concepción, la Comisión Europea (2011): planteó que para cumplir con la responsabilidad social se exigen nuevas capacidades y cambios en los valores y los comportamientos. significa, que los centros educativos al integrar la responsabilidad social se crea un desarrollo sostenible y se forman ciudadanos responsables en los programas educativos pertinentes, lo que requiere de mayor compromiso para lograr equipos de docentes investigadores desde su hacer en el aula como en la interacción en conjunto con la comunidad en general.

Desde ese escenario, se observa en las instituciones públicas diferentes situaciones sobre el conocimiento práctico que implica la 
responsabilidad social para la integración de todos los actores llámense escuela y comunidad. Es decir, que todos deben participar en el que hacer educativo para la transformación de su realidad, la cual implica una praxis comprometida y en correspondencia a la formación critica socio educativo.

\section{Teoría y Conceptos}

\subsection{Propósito General}

Comprometer socialmente al personal directivo- docente para fortalecer el entorno comunitario de la UEN Héroe Nigale del municipio Valmore Rodríguez del Estado Zulia.

\subsection{Descripción del Escenario}

La investigación se desarrollara en el Municipio "Valmore Rodríguez del Estado Zulia, específicamente en el sector "La Playa", muy concretamente, en la Escuela Básica Nacional Héroe Nigale la cual está ubicada en el sector la playa carretera los Teques limitando con los Teques, rio Misoa, sector curva el coquito, y el lago de Maracaibo, atendiendo una matrícula de ciento ochenta y dos (182), distribuidos de la siguiente manera: 39 estudiantes de educación inicial, 70 en educación básica bolivariana, 73 de educación básica, media general y diversificada, está distribuida en tres niveles o modalidades atendiendo los turnos matutino y vespertino. Por otra parte, en este tipo de institución reciben una alimentación balanceada, la cual contempla desayuno, almuerzo y merienda se rige por el programa de alimentación escolar del gobierno bolivariano implementado con la finalidad de mejorar la calidad de vida de los estudiantes de entornos, es decir; sus familias cuentan con varios recursos económicos debido a la carencia de fuente de empleos formales, la mayoría trabajan en el comercio informal pescando y casas de familias. 


\subsection{Percepción de la Investigadora}

En la visita realizada a la Escuela Básica Nacional Héroe Nigale se constató que es una institución localizada en el área rural del Municipio Valmore Rodríguez, la cual a través de los años se ha convertido en una escuela importante para el proceso de formación y un punto central para toda la comunidad.

En este sentido, los nuevos lineamientos enmarcados en el documento "líneas estratégicas en el marco del proceso curricular venezolano del Ministerio del Poder Popular para la Educación (2014): como ente rector en materia educativa, la cual, exige compromiso del director y del docente para el alcance de las metas educativas y para lograr el interés no solamente del estudiante sino de los padres y representantes con respecto al proceso de aprender - aprender; articulados en el líneas estrategias que conlleva a la formación integral del estudiante donde el rol del director y del docente es transcendental en la transformación social que exige compromiso y responsabilidad con la humanidad..

Desde esa perspectiva, el director y los docentes en la praxis diaria, son el esfuerzo sostenido para que trascienda en los espacios escolares, estrategias metodológicas de aprendizaje y enseñanzas pertinentes, contextualizadas e innovadoras que desarrollen habilidades que facilitan el análisis reflexivo y crítico del contexto nacional e internacional y aporten a la construcción de una pedagogía propia.

Partiendo de ese escenario, se evidencia en la escuela Héroe Nigale que el personal directivo - docente manifiestan apatía sobre el compromiso social de dicho procesos, con respecto a las políticas educativas, planes de la nación, cumplimiento de la articulación escuela, familia, comunidad, formaciones permanentes, formas de contribución de los docentes -director con la comunidad del entorno, entre los fines del estado y las concreciones en la actividad escolar; de transformación de la institución educativa, el logro de 
la pertinencia social, mediada por la participación de todas y todos, responsables y corresponsables de la educación.

Es decir, se dedica a cumplir con los contenidos exigidos, y la mayor ocupación está referida a las exigencias administrativas del MPPP sin importarles los problemas de la institución, o las situaciones en donde se observa problemas de tipo familiar, maltrato verbal, por nombrar alguno de los tantos hechos problemáticos presentes en la escuela, Porque según. MPPE en las líneas orientadoras de acción para la gestión escolar sugiere la corresponsabilidad con la integración y conformación de comunidades educativas tales como (estudiante, representantes, obreros, administrativos, padres - representantes, comunidades organizadas) con el fin de compartir, saberes, conocimientos, desde el dialogo, reflexión, análisis, críticas, síntesis creativas; para construir subjetiva con compromiso, postura ética, conciencia social. Para dar respuesta a las necesidades pedagógicas, socio productivas, cultural, científicas, tecnológicos de la institución y la comunidad.

Es necesario acotar que dicha evidencia trae como efecto que el personal directivo -docente muestran desmotivación para las formaciones permanente ya que las mismas no están acorde con el horario establecido, al mismo tiempo manifiestan que la mayor ocupación está referida a las exigencias de los contenidos curriculares y administrativas del MPPP.

En tal sentido esto trae como consecuencia que la educación para el futuro no logre superar las debilidades existentes en el sistema educativo, así como la educación de calidad que se quiere, para alcanzar transformación social. Es por ello que, para analizar las causas y efectos de un problema se procedió hacer uso de una herramienta importante como lo es el árbol del problema, este permite visualizar la realidad de una forma abierta y comprensible. Al implementarse permite obtener información acerca de cómo los integrantes del grupo de investigación visualizan los problemas de la 
institución educativa, lo cual le permite a la investigadora otros puntos de vista sobre la organización educativa.

\section{4. Árbol del problema}

Según, Bobadilla (2010), expone el árbol del problema es una herramienta que se utiliza para la identificación y el análisis de las causas más relevantes. Este sentido el árbol del problema se enfoca en la forma de percibir la situación de la institución educativa como un sistema con vida propia, con relaciones estrechas entre todas sus partes para funcionar adecuadamente, se comparan con las partes con las funciones, anotando lo visualizado por los participantes, para cada elemento constituyente del árbol social, como se muestra en el cuadro 1.

Cuadro 1. Árbol del Problema.

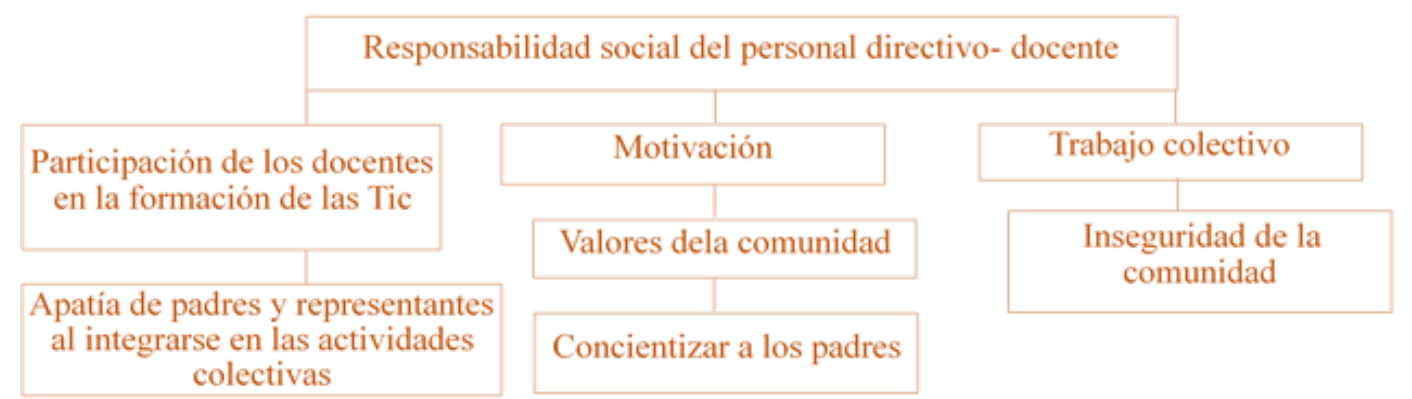

Fuente: La Autora (2015).

\subsection{Idea general de la investigación.}

El mundo de la era globalizadora plantea retos y exigencias a las organizaciones educativas, tanto en la participación como en la colaboración, surgen desafíos cada vez más acuciantes en la sociedad actual, por ello, el futuro de la educación pública está orientado a la implicación de los centros educativos en sus comunidades socioculturales, considerando la necesidad de unir esfuerzos para obtener más fácilmente el objetivo común, además de 
darle a cada uno la oportunidad de ejercitar sus competencias e iniciativas asumiendo activamente sus responsabilidades y compromisos.

A esto se le acuña el criterio de Certo (2001): donde plantea las empresas deben ejecutar actividades de compromiso social porque la rentabilidad y el crecimiento van de la mano con un tratamiento responsable de los empleados, los clientes y la comunidad. En esencia, implica ejecutar las actividades de compromiso social, esta es una forma de ganar mayor utilidad organizacional.

Sobre la base de la consideración anterior el compromiso social de las instituciones va más allá de obtener provechos, se hace necesario mencionar que, según Martínez, Krichesky y García (2010): uno de los factores claves de las instituciones educativas para su buena organización y funcionamiento, es la existencia de equipos directivos y docentes comprometidos con la educación, son los que constituyen el eje central para la educación de calidad.

Por otra parte, en América Latina y el Caribe el compromiso del personal directivo - docente para la educación es vista como la transformación a favor de la superación social de las instituciones educativas emblemático, las Políticas para el Cambio y el Desarrollo de la calidad educativa, donde el énfasis es el ejercicio pleno de la ciudadanía, la equidad social, y la étnica.

Dentro de este marco la educación Venezolana ha generado transformaciones con relación al sistema educativo donde el director- docente adquiera compromiso con las organizaciones educativas, cumpliendo un rol que responda a los planes de la nación referidos al ámbito educativo, es decir implica llevar la escuela hacia las comunidades brindando desde la formación académica apoyo y orientación en la resolución de problemas confrontados en la gestión escolar, lo que requiere una educación comprometida con las ideas democráticas, de libertad e igualdad como elemento que logre incorporar en la ciudadanía la idea y el compromiso del trabajo productor como elemento que humaniza al hombre. 
Asimismo, el sistema educativo requiere el manejo de los procesos de interrelación y comunicación que lleva a cabo el director- docente, para mantenerse en contacto cercano la permanente con todos y cada uno de los actores que participan en el quehacer de la escuela.

Álvarez (2010): asumiendo la praxis diaria del director - docente, como un esfuerzo sostenido que trascienda en los espacios escolares, estrategias metodológicas de aprendizaje y enseñanza pertinentes, contextualizadas e innovadoras donde se desarrollen habilidades que faciliten el análisis reflexivo, crítico del contexto nacional e internacional que aporten a la construcción de una pedagogía propia.

En este mismo orden de ideas, se plantea que el compromiso social de la institución educativa va más allá del cumplimiento del currículo, reglamentos o normativas del Ministerio del Poder Popular para la educación (2014): sino que contempla el ejercicio de la ciudadanía desde la perspectiva ética, en sus acciones cotidianas, dándole rasgos de identidad significativos que hablan de una organización con valores, sobre todo con sensibilidad social para construir el tejido social de una sociedad más equitativa

De igual forma, Fuentealba y Imbarack (2014): refieren que el personal directivo - docente está en correspondencia con el compromiso ante la sociedad educativa donde se reproducirán y desarrollarán los conocimientos, competencias, valores necesarios para una satisfactoria vida económica, social, y personal. Quiere decir que el personal directivo - docente no solo debe cumplir con sus funciones, sino ir más allá para la integralidad social de todos los actores del hecho educativo.

Atendiendo lo anterior, el Ministerio del poder Popular para la Educación contemplado con el artículo 5 de la Ley Orgánica de Educación (LOE, 2009): aspira que el personal directivo- docente este centrado en el desarrollo de la sociedad donde los esfuerzos para una humanidad deben ser responsable, equitativa, justa, enfocados a construir ciudadanos para un país, capaces de 
ser, de aprender, de vivir y convivir con otros en el mundo, en un espacio que deje de ser un grupo para ser de todos y con todos.

A esto se le acuña, que las instituciones educativas deben mantener una estrecha vinculación con el entornos comunitarios, para así darle cumplimiento a los planes de la nación, actuando de manera reflexiva crítica ante el hecho educativo, no solo cumplir con los contenidos curriculares, y exigencias administrativas sino que mantenga un mira hacia los paradigmas, sostenidos hasta ahora acerca de la imposibilidad de realizar cambios desde espacios geográficos olvidado de los grandes programas sociales.

Es preciso señalar que el Ministerio del Poder Popular para la Educación (2014): propone que las Escuelas Bolivarianas deben asumir como convicción el compromiso y responsabilidad para la transformación en materia educativa con la participación de todos los actores del proceso, considera considerando que las mismas son transformadora de la sociedad en el cual se concrete e identifique con identidad nacional, práctica de participación, formación crítica, integración a la comunidad, el arraigo de la historia, costumbres, valores, apuntan a la construcción de una sociedad más justa, fortaleciendo a las persona, comunidades para que sean protagonistas de la transformación del país.

Cabe resaltar que a los directores- docentes han asumiendo el compromiso con las organizaciones educativas y la misma comienza a cumplir un rol que responda a los planes de la nación referidos al ámbito educativo, es decir implica llevar la escuela hacia las comunidades brindando desde la formación académica apoyo y orientación en la resolución de problemas confrontados en la gestión escolar, lo que requiere mayor compromiso para lograr equipos de docentes investigadores de su hacer educativo en conjunto con la comunidad e institución en general.

Es por ello que, la presente investigación tiene como propósito comprometer socialmente al personal directivo- docente para fortalecer el 
entorno comunitario de la UEN Héroe Nigale del municipio Valmore Rodríguez del Estado Zulia. En este sentido, las acciones estarían dirigidas a realizar verdaderas transformaciones partiendo de las necesidades de los grupos comunitarios emergentes de los entornos comunitarios circundantes de las instituciones educativas; y los resultados enfocados en la acción participativa proveniente de la implementación de la acción transformadora con la finalidad de evaluar, reflexionar acerca de esos hallazgos, y realizar nuevos planes de acción que permitan promover cambios significativos a la institución.

\subsection{Fundamentos Teóricos}

\subsubsection{Responsabilidad Social}

Howard (2016): plantea que la responsabilidad social empresarial es definida como las obligaciones de los empresarios para impulsar políticas corporativas para tomar decisiones o para seguir líneas de acción que son deseables en términos de los objetivos y valores de la sociedad, señala que la responsabilidad social se trata acerca de la idea de que su aceptación voluntaria por parte de los empresarios en el largo plazo, pueda repercutir en una disminución de los problemas económicos y una mayor posibilidad de alcanzar los objetivos de una organización empresarial.

Cabe destacar que desde las instituciones educativas cumplen una función pública, la de educar, la cual le genera una responsabilidad social en lo relacionado con la formación del estudiante. Por lo tanto, los gerentes del sector educativo pueden utilizar diferentes modalidades para incluir en las labores cotidianas la responsabilidad social en sus estudiantes, docentes y demás personal cumpliendo con las expectativas deseadas por la sociedad.

Actualmente existen la figura de colectivo institucional los cuales consisten en un grupo elegido democráticamente para fomentar en los miembros de la comunidad de la cual hace parte la institución educativa para integrarse en la institución y apropiándose de la formación necesaria para 
conocer los contenidos curriculares, conocer las diferencias, contribuir con el mantenimiento de la escuela ejerciendo la participación ciudadana desde la tribuna de los encarados de la formación de ese ciudadano honesto, solidario y equitativo.

Al respecto es necesario mencionar la ausencia de un verdadero compromiso y responsabilidad con las instituciones educativas en todos los actores del hecho educativo, todo se queda en propuestas sin accionar de los directores, docentes, representantes y comunidad dejando la responsabilidad que deberían estar compartidas por todos los interesados en mejorar la calidad de vida de todos los habitantes de sus comunidades. Además, es necesario que el compromiso y la responsabilidad social con la institución no se queden en una asistencia pasiva a las asambleas convocadas por la escuela, sino que exista la participar para la resolución de los problemas ya que la educación futura de los niños, niña y adolescente es responsabilidad de todos los actores educativos.

\subsubsection{Compromiso Social}

Según Martínez, Krichesky y García (2010): el compromiso social es un método de acercamiento y circulación por los aportes posibles en el interior del campo de juego en el que se inscribe el sector, y que cada sujeto se acerca a configurar su propio campo de posibilidades de manera no mecánica ni predeterminada, sino bajo la influencia de su capital cultural, su historia personal, el momento histórico que vive. Por otra parte, el autor refiere el compromiso radical de la escuela con la educación del ser humano no puede eludir su posición crítica con las políticas de injusticia y desigualdad. Ésta debe seguir siendo una cuestión básica en todo educador.

De igual forma, García (2012): el compromiso social es un elemento que

refiere a funcionar agregando valor a sus entornos comunitarios con respecto a la institución, esto implica por parte de las instituciones en este caso la 
escuela que debería reflexionar acerca de su trabajo, cual es el impacto que tiene en la comunidad, como contribuye al desarrollo endógeno de la misma y de la sociedad en general.

\section{Metodología}

Para el desarrollo de este artículo, la población objeto de estudio es aplicada 17 docente, 1 subdirector y 82 representantes. Seleccionando la investigación-acción participativa apoyada en Elliont (2007): que expresa que la investigación-acción se ejecuta paralelamente con el esparcimiento del conocimiento científico y la solución de un problema, mientras se desarrolla, las competencias de sus referidos participantes (sujetos e investigadores) y es llevada a cabo en colaboración de todos los involucrados, en una situación concreta, a su vez realimentando la información en un proceso cíclico. Este modelo está relacionado con las técnicas utilizadas tales como la observación, la entrevista, grupo focal, que permitió el análisis e interpretación de los resultados, a través del registro de campo como instrumento utilizado, que permitió las acciones transformadoras colectivamente con la participación de los involucrados en la problemática, donde actúen toda la institución en una continua reflexión sobre la praxis diaria.

\section{Análisis de los Resultados de la Categorización}

Durante el desarrollo de las acciones, se logró receptividad y la participación corresponsable del personal directivo- docente, padres y representantes la cual se sintieron motivados para involucrarse con todos los actores socio educativos, quienes tuvieron la oportunidad de expresar sus experiencias y compartir ideas, propiciando la interacción transformando posturas y comportamientos ante quienes tienen la responsabilidad de formar los ciudadanos, ciudadanas del mañana. 
Asimismo, los aportes constituyeron a que se debe afianzar la planificación de proyectos con autores como Morín (2010): que conduzcan hacia la educación del futuro donde se exija un compromiso de todos y cada una de las personas que hacen vida en la institución educativa.

Aunado a lo anterior las acciones ejecutadas ayudaron al personal directivo- docente de la escuela a programar actividades y a su vez aplicar estrategias para la participación e integración de los padres y representantes a la institución en mejora de algunos problemas que atañe en proceso de aprendizaje de los estudiantes.

$Y$ finalmente se obtiene que en conjunto trabajaran por un solo fin, educación de calidad para un futuro próximo, dejando claro que la educación no se basa solamente en el cumplimiento del currículo emanado por el Ministerio de Educación sino de las verdaderas necesidades del entorno comunitario.

\section{Conclusiones}

Considerando el propósito se llegó a lo siguiente que se debe reflexionar profunda en el cambio de actitud del director- docentes, padres, representantes, comunidad para asumir un verdadero compromiso y responsabilidad social para la transformación del modelo educativo, para la educación del futuro de la Unidad Educativa Nacional Héroe Nígale teniendo como propósito la integralidad, desarrollo y participación activo de todos los actores socioeducativos, de igual forma, es necesario incluir la transdisciplinariedad en los planes de estudios si se desea para formar personas competentes, cooperativas, criticas, democráticas, con la finalidad de la comprensión del hombre en la interacción con el mundo.

Así mismo, tomar la integración de saberes, métodos, valores y principios, brindando la oportunidad al ser humano de seguir estudiando a lo largo de su vida, aprender de diversas formas y desde diferentes fuentes 
(teóricas, tecnológicas, otras). Igualmente, que se desarrolle en las instituciones educativas la actitud desde el autoaprendizaje, para adaptarse a un tiempo de cambios permanentes, y así poder comprender el mundo donde le toca vivir, atravesando barreras, cultura, idiomas, sociedad, donde se rompan paradigmas, y se deje de lado las ideas antiguas que no han dado resultados favorables, para dar paso a cambios significativos.

\section{Referencias}

Álvarez, M. (2010). Liderazgo compartido: Buenas prácticas de dirección escolar. Las Rozas: Wolters Kluwer España.

Bobadilla, P. (2010). El árbol Problémico. Perú: PACT/PERÚ.

Comisión Europea (2011). Comunicación de la comisión al parlamento europeo, al consejo, al comité económico y social europeo y al comité de las regiones. Bélgica.

Certo, S. (2001). Administración moderna: diversidad, calidad, ética y el entorno global. 8a edición. Colombia: Pearson Educación.

Elliont, G. (2007). Desafíos de la Investigación Cualitativa Catedrática de Pedagogía Social. Universidad Nacional de Educación a Distancia (UNED). Chile.

Fuentealba, R., y Imbarack, P. (2014). Compromiso docente, una interpelación al sentido de la profesionalidad en tiempos de cambio. Estudios Pedagógicos (Valdivia), 40(Especial), 257-273, eISSN: 0718-0705. Chile.

García (2012). Un compromiso de los actores socioeducativos para cambiar las escuelas. Congreso Internacional de Transformación Educativa.

Howard, R. (2016). Responsabilidad social de la empresa. Washington, Estados Unidos.

LOE (2009). Ley Orgánica de Educación. Gaceta Oficial № 5.929 Extraordinario del 15 de agosto. República Bolivariana de Venezuela: La Asamblea Nacional.

Martínez, C., Krichesky, G., y García, A. (2010). El orientador escolar como agente interno de cambio. Revista Iberoamericana de Educación, 54(1), 107-122, e-ISSN: 1022-6508. España.

Ministerio del Poder Popular para la Educación (2014). Líneas estratégicas en el marco del proceso curricular venezolano. Venezuela.

Morín, E. (2010). Los siete Saberes Necesarios para la Educación del Futuro. Publicado por Vallejos. UNESCO. 


\section{Marilú del Valle Parra Rojas \\ e-mail: mvlu27@gmail.com}

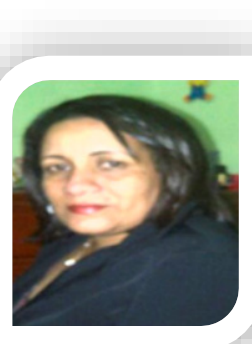

Nacida en Ciudad Ojeda estado Zulia, Venezuela. Cursó estudios de pregrado en la Universidad Nacional Experimental Rafael María Baralt obteniendo título de Lcda. Educación Integral, posteriormente realizó estudios de postgrado en Evaluación por la Universidad Valle del Momboy, estado Trujillo, es Cursante del Doctorado de Educación de la Universidad Nacional Experimental Rafael María Baralt, estado Zulia, y actualmente se encuentra en espera del título como Magister en Gerencia Educativa de la Nororiental Gran Mariscal de Ayacucho. En la actualidad se desempaña como Subdirectora de la Unidad Educativa Nacional Bolivariana Daniel Mavarez, con 13 años al servicio del Ministerio del Poder Popular para la Educación.

El contenido de este manuscrito se difunde bajo una Licencia de Creative Commons ReconocimientoNoComercial-Compartirlgual 4.0 Internacional 